\title{
H2020 OSMOSE Project: the Italian demonstrator. Testing flexibilities resources in a coordinated approach
}

\author{
Luca Orrù, Leonardo Petrocchi, Alessio \\ Siviero, Francesco Silletti, Giuseppe \\ Lisciandrello, Gino Albimonti. \\ Terna S.p.a. \\ Rome, Italy
}

\author{
Dario Ronzio, Ilaria Losa \\ RSE S.p.a. \\ Milan, Italy
}

\author{
Marilena Lazzaro \\ ENGINEERING Ingegneria Informatica S.p.a. \\ Rome, Italy
}

\begin{abstract}
Among the many "low carbon electricity" initiatives launched by the Horizon2020 programm, the OSMOSE ${ }^{1}$ project aims to assess all possible ways to provide flexibility to the European power system. This article provides a brief explanation, in terms of organization and technological implementation of the Italian demonstrator (Work Package five) belonging to the OSMOSE project.
\end{abstract}

Index Terms - Demand Side Response - Dynamic Thermal Rating - Flexibility - Smart grid - Synthetic Inertia

\section{INTRODUCTION}

Osmose is a H2020 EU founded project that aims at developing innovative solutions to enhance transmission grid flexibility in order to better integrate Renewable Energy Sources (RES). OSMOSE adopts a global approach that address the increasing need of flexibility services in the system (e.g.: improved balance of supply and demand in electricity markets, provision of existing and future system services and allowance of a dynamic control of electricity flows) and assess the local availability of different sources of flexibilities (RES, demand-response, grid and new storages). In order to prove the viability of the proposed approach in real operating conditions, four demonstrators have been included in the project, leveraging on the cooperation of four European Transmission System Operators: RTE (France); REE (Spain), Eles (Slovenia) and Terna (Italy). This paper describes the features and innovations of the Italian demo.

\section{STRUCTURE OF THE ITALIAN DEMO}

The Italian demo aims to demonstrate multiple grid services provision from Renewable Energy Sources and Demand-Side Response (DSR) coordinated through a smart management system, by addressing these objectives:

- Use Case 1: to improve congestion management on $\mathrm{HV}$ grids and to maximize RES production by

\footnotetext{
${ }^{1}$ Optimal System-Mix Of flexibility Solutions for European electricity grid
}

coordinated use of Dynamic Thermal Rating (DTR) short-term forecasts and DSR

- Use Case 2: to demonstrate, in a relevant HV grid area, the reliability of provision of Synthetic Inertia (SI) and Automatic Voltage Control (AVC) provided by single large wind power plant

- Use Case 3: to demonstrate, in a relevant HV grid area, the reliability of provision of Frequency Restoration Reserve (FRR) and AVC by a single or by a few aggregated large industrial loads

The demo area was selected by identifying a grid portion in which congestion issues have been relevant and thus the test of flexibility services provision would have been the most effective. According to Terna's data, congestions occur with a significant frequency on $150 \mathrm{kV} \mathrm{HV}$ lines between Apulia and Basilicata regions (Figure 1), causing a curtailment of $1.1 \%-1.3 \%$ of total national wind power production (data referring to Terna analyses performed in 2017, during the project design phase). This area is also affected by problems caused by reversed energy flows in some HV/MV substations for several hours of the year (approximately from $1 \%$ to $5 \%$ of the time). In the same area, there is also a so-called essential coal power plant, which is kept in operation for voltage stability needs. Overall, the demo includes:

- 7 main $150 \mathrm{kV}$ lines as the test field;

- 2 Wind power plants, (included in Use Case 2);

- 7 industrial loads, (included in Use Case 1 and 3).

The demo activities will last for approximately 10 months. Test results will be available by the end of 2021 and should highlight which innovation solutions could help the TSOs to solve congestions and reduce RES curtailment by means of sustainable resources. 


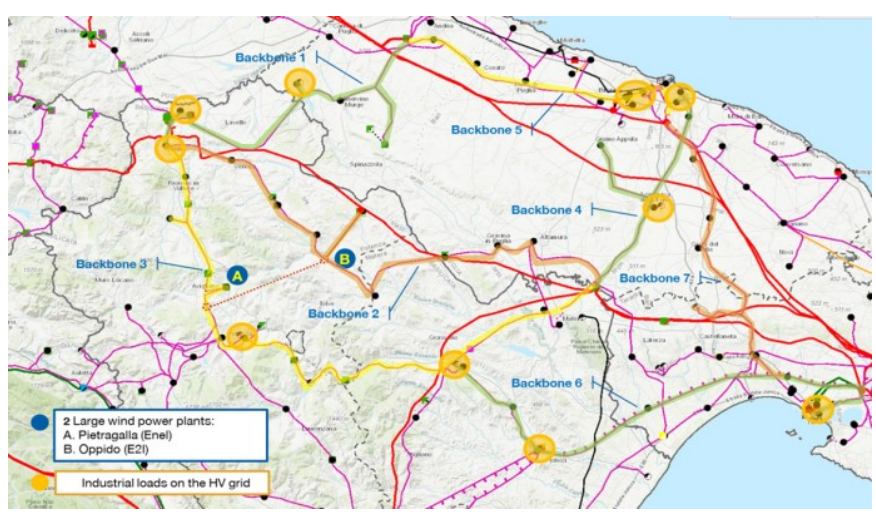

Figure 1. Demonstrator area and flexibilities resources

The Italian demo involves partners belonging to the whole value chain of energy system: Utilities (Terna), R\&D center and universities (ENSIEL, RSE), industrial providers (ABB, IBM, ENGINEERING), flexibilities service providers (ENEL, COMPENDIA, EDISON).

\section{SOFTWARE DEVELOPED}

The Zonal Energy Management System (Z-EMS), developed by IBM is the main software of the demo, aiming at detecting and solving future congestions in the sub-transmission grid. The Z-EMS solves an Optimal Power Flows in the 150 and $132 \mathrm{kV}$ grid, in order to minimize costs and future congestions, with 3 hours ahead time horizon and 15 minutes time resolution. The operation of the Z-EMS relies on the following innovative flexible tools: the Dynamic Thermal Rating and the Demand Side Response. Input data needed by Z-EMS are: programmable generation forecast, photovoltaic and wind generation forecast, load forecast from PREVEL, load flow outputs, DTR loadability curves, data exchanged with DSR resources. These data are provided by the Hand of Data (HoD), a software component that manages the data orchestration. The HoD, developed by ENGINEERING, brings together siloed data from multiple data storage location and systems, combining and organizing it, and making it available for data analysis and processing tools. Therefore the HoD, is the component responsible to retrieve and transform data, to activate the Z-EMS and to manage its output. The HoD connects the Z-EMS to the different data sources involved in the demo including the systems adopted by TERNA for retrieving the programmable generation forecast, photovoltaic and wind generation forecast and the PREVEL for the provision of load forecast. Data are retrieved using different protocols and they are turned to a standard format compliant to the Z-EMS requirements. This transformation allows to speed up the process of data analysis since the Z-EMS does not need to spend time reconciling data. Moreover, the HoD works as contact point between the DTR tools deployed on the Master Nodes and the Z-EMS and it can easily access to the DTR loadability curves. The Dynamic Thermal Rating tools, in turn relies on the HoD for the provision of data input at the basis of their own elaboration. One important step of the data orchestration is the activation of Z-EMS: once all inputs are available, the ZEMS starts its execution. The Z-EMS outcome is stored by the HoD in the "Share Z-EMS", the ICT solution provided by TERNA to collect all demo relevant data in real time. The $\mathrm{HoD}$ arranges the data orchestration through a scheduling processing that handles the timing of overall demo execution. This process takes in account of the specific security levels required by the TSO to guarantee that all the requirements related to data protection and data integrity are met. Indeed, a specific architecture has been set up for the demo realization (Figure 2)

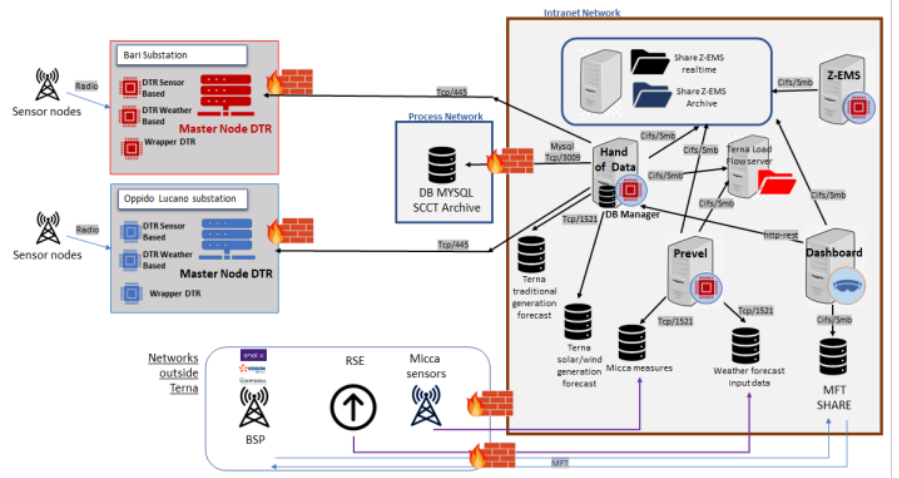

Figure 2. ICT Architecture of the Italian demo

PREVEL is the load forecast algorithm, installed and configured within Terna ICT infrastructure, developed by RSE. PREVEL permits to calculate, every quarter of hour, active and reactive power forecasts for all electrical loads connected to 150 and $132 \mathrm{kV}$ grid in the demo area (Figure 3 ). Indeed, the algorithm is applied to border lines, assuming that their behaviour is similar to the behaviour of loads.

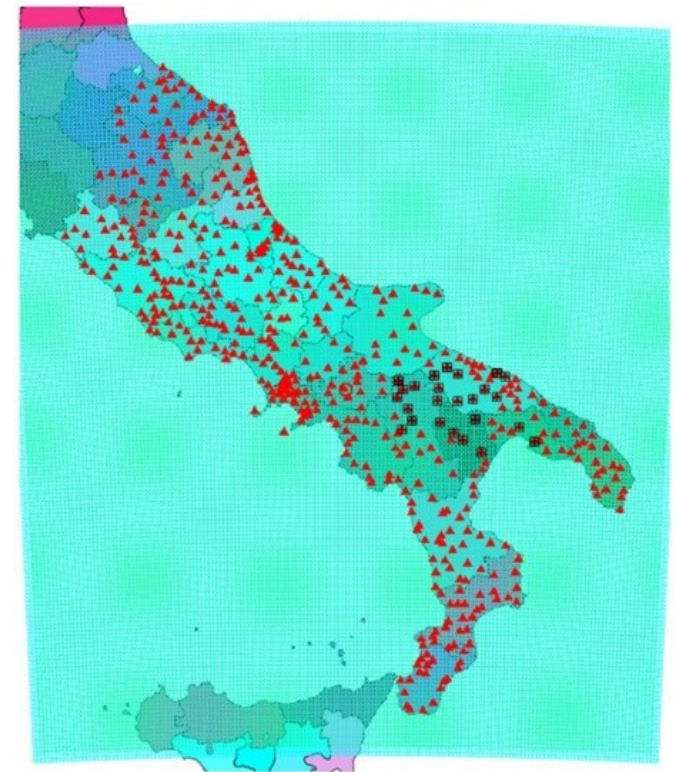

Figure 3. Geographical position of electrical loads to be forecast by PREVEL.

PREVEL provides the forecasts of some meteorological variables required by the DTR systems, and electric loads. For both cases the output of two different numerical weather 
prediction models, i.e. the Weather Research and Forecasting Model [1] and the Regional Atmospheric Modelling System [2] driven by the initial and boundary are provided by the Integrated Forecast System, developed by the European Centre for Medium Range Weather Forecasts, UK, and the Global Forecast System, developed by the National Oceanic and Atmospheric Administration, USA. The four outputs are combined in order to obtain an optimized forecast for each weather variable. The meteorological data required by DTR systems (wind speed and direction, temperature, global irradiance, relative humidity) are obtained adjusting the weather forecasts taking into account the corresponding time series acquired in real-time by means of an autoregressive model with exogenous inputs (ARIMAX [3]). In this case a training period of 10 days is used. Figure 4 shows input data of PREVEL and its output with respect to the loads forecast.
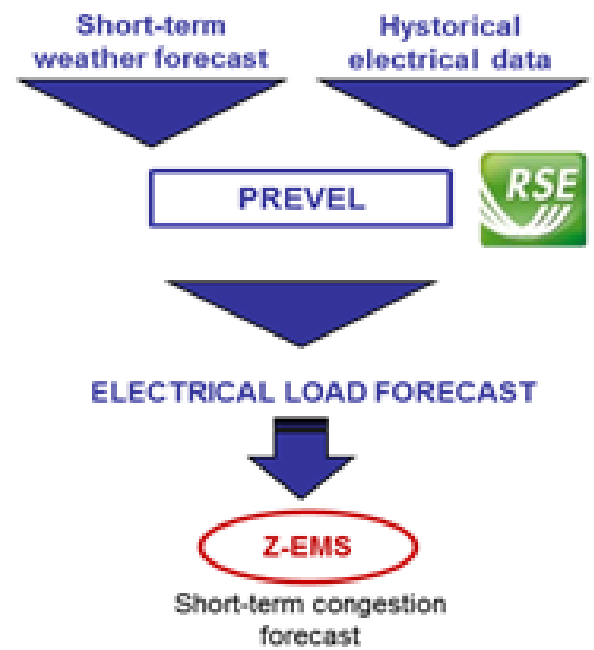

Figure 4. PREVEL data flow for electrical load forecast

The load forecasts are performed in two steps. First, the short-term forecasts are computed for each electrical load using a supervised machine learning approach, more precisely a Random Forest (RF, [4]) algorithm trained with weather variables provided by the optimization described previously (air temperature, relative humidity, wind speed at 10 and $75 \mathrm{~m}$, global, direct and diffuse irradiation, astronomical angles), and calendar factors (day of the week, such as public holiday working day, bridge or holiday). The $\mathrm{RF}$ was implemented by means of the package ranger [5] of the statistical library $\mathrm{R}$, and both model creation and prediction were parallelised to reduce CPU time. The operational configuration foresees a training dataset of at least 6 months, updated every week. The second step provides the very-short term forecast obtained by applying an ARIMAX fed by real-time electric load measures and by using the short-term forecast as exogenous input. The training set is 10 days long, updated every 15 minutes. Figure 5 shows a comparison between the forecasted and measured electrical for two case studies: $29^{\text {th }} \div 30^{\text {th }}$ June and $29^{\text {th }} \div 30^{\text {th }}$ September 2020. The Pearson's correlation $\mathrm{R}^{2}$ is 0.942 for the very-short term, and 0.829 for the short term forecast, whilst the percentage absolute error (mean of absolute difference between forecast and measure, divided by the $99^{\text {th }}$ percentile of the measure) increases monotonically with time horizon from $0.59 \%$ at $+15^{\mathrm{m}}$ to $2.03 \%$ at $+180^{\mathrm{m}}$ (red line in the inset of Figure 6).

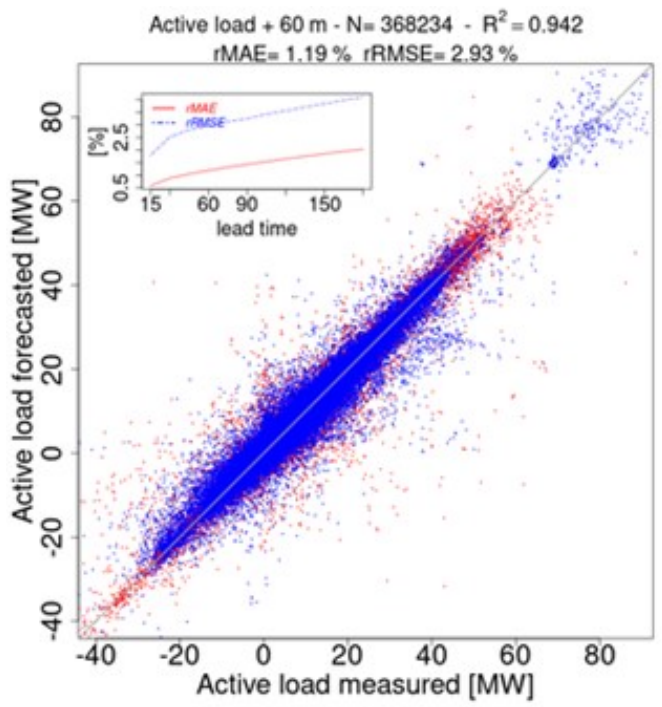

Figure 5. Scatter plot of active electric power forecasted during 29th - 30th June (red points) and 29th - 30th September (blue points) of 2020. The forecast horizon is 1 hour ahead. The inset shows the percentage absolute error (rMAE, in red) and the quadratic one (rRMSE, in dotted blue) with respect to leadtimes

\section{INNOVATIVE DYNAMIC THERMAL RATING (DTR) APPROACHES}

Two DTR methods developed by Ensiel [6] are tested: i) a Sensor-Based Method, based on a self-organizing sensor network composed by cooperative Smart Nodes deployed along the line route and connected via radio to Server Master Nodes, and ii) a Weather-Based Technique, based on a thermo-mechanical model of the monitored line. The SensorBased Method (Figure 6) foresees the installation of 6 Weather Sensor Stations (for the measurement of environmental temperature, humidity, wind speed, solar radiation), 2 Micca Sensors (aimed at the calibration of the method's algorithm) involving 6 pylons of 2 transmission lines in the South of Italy (3 Stations and 1 Micca Sensor per line) and 2 PCs (Master Node Servers located in the relative substations), creating a distributed approach, in order to monitor the weather conditions on the entire line and being able to find out which is the most critical span. The installed Sensor nodes communicate via radio with each other and with the dedicated Master Node, consisting of a PC installed in the relative substation aimed to calibrate the model of temperature prediction by comparison with the Micca measure; the output of the complete system is the loadability curve, which indicates how long a current value can be sustained, even higher than the nominal one. Weather forecasts are used to extend the result to a time horizon of 3-6 
h. Once calibrated the algorithm, this method does not require to install further Micca Sensors for the DTR. This advantage lows to avoid the need to install other expensive devices.

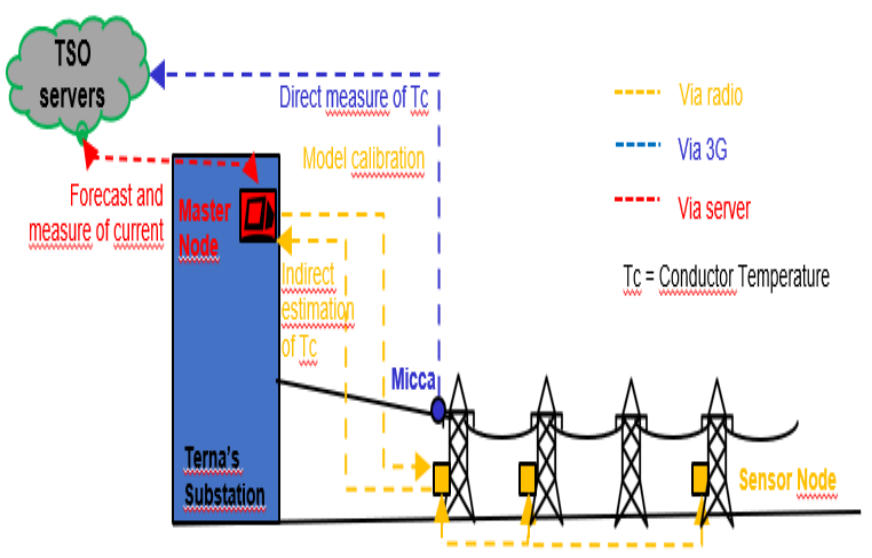

Figure 6. Sensor based DTR Method installation pattern [6]

The Weather Based Method (Figure 7) requires to collect several information that the thermo-mechanical parameters of the line; however it does not require to gather further measurements from the field. In fact the algorithm uses weather forecasts (provided by RSE developed models) and power flow forecasts (provided by Terna) as input data, and it estimates the loadability curve of the line. This method requires no installation of sensors, thus allowing significant savings in money and time.

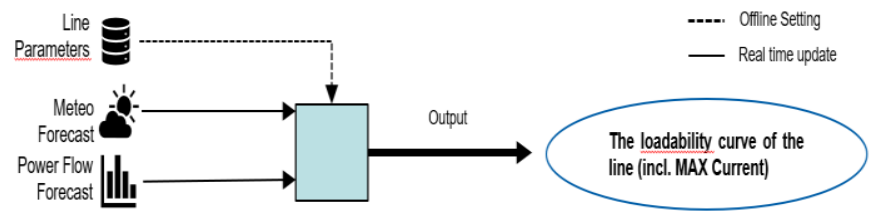

Figure 7 - Weather based DTR Method pattern

The comparison of the results given by the two tested methods is also one of the targets of the project itself.

\section{FLEXIBILITY FROM INDUSTRIAL LOADS THROUGH DEMAND SIDE RESPONSE (DSR)}

Seven industrial loads, connected to the high voltage grid and located in the demo area, will be tested to provide different flexibility services through DSR, including both active and reactive power services. The loads included in the demo belong to different areas of the industrial sector such as: oil refinery, car manufacturing, foundry, energy supply, steel factory and a Military site. All these loads will be managed by three different project partners, Compendia, Edison and EnelX, the Balance Responsible Parties (BSP). The role of a BSP is to act as an interface between the industrial plants and
TSO's requests, in order to enable the industrial load to provide significant grid flexibility services. During the tests, Terna will send a regulation request based on the availability previously communicated by aggregators. In a second stage, the BSPs handle their resources in order to fulfil the flexibility request submitted by TERNA. The industrial load that accepted to join the Italian demo were subjected to a flexibility audit that aimed at enabling the different loads to provide the requested grid services. ABB then realized seven different implementation projects. The ABB analysis aimed to install the equipment needed to enable the flexibilities identified. According to the energy audit results (Figure 8), the following flexibility services will be tested:

- Congestion resolution, in coordination with the output of the Z-EMS algorithm, for a maximum theoretical regulating capacity of $121.3 \mathrm{MW}$ (27.3 MW loads, 94 MW generators)

- Provision of Frequency Restoration Reserve (FRR), by automatic active power regulation, for a maximum theoretical capacity of $94.5 \mathrm{MW}(500 \mathrm{~kW}$ loads, $94 \mathrm{MW}$ generators)

- Voltage regulation (AVC), by the provision or the absorption of reactive power, for a maximum theoretical capacity of 34 MVAr (3.5 MVAr loads, 30.5 Mvar generators)

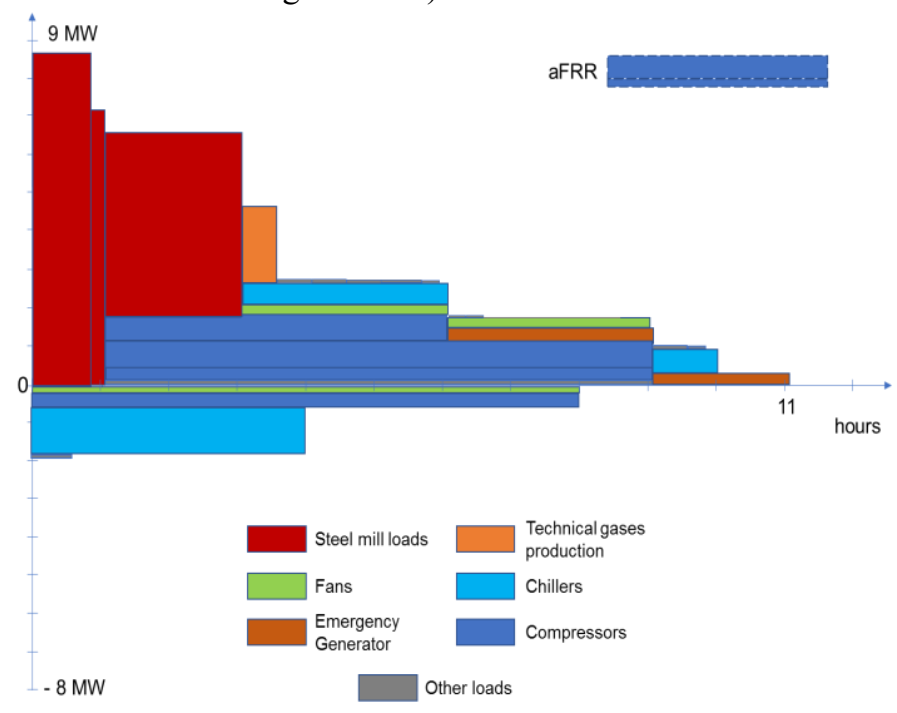

Figure 8. Overall flexibility aggregation example

The actual capacity of the resources will be assessed during the tests phase and it will depend both on the operating energy conditions and on plant production processes.

\section{FLEXIBILITY FROM RES POWER PLANTS}

The experimentation of innovative grid services with RES and storage technologies is one of the OSMOSE project targets. The Enel Green Power (EGP) integrated wind and storage power plant in Pietragalla and the Energie Speciali (E2i) wind power plant in Vaglio will be the testing grounds for the experimentation: the EGP power plant is made of a 18 
MW wind power plant and a $2 \mathrm{MW} / 2 \mathrm{MWh}$ Li-ion Battery Energy Storage System, while the E2i plant consists of a total $35 \mathrm{MW}$ wind turbines (2.5 MW each). The services to be tested are automatic voltage regulation (AVC), which, as of today, is only provided by conventional thermoelectric power plants, and synthetic inertia, which aims to mimic the inertial response provided by rotating heavy masses connected to the grid. In particular, the experimentation on AVC aims at testing both "local" and "centralized" voltage control and therefore the plants will receive and act according to either a tension or reactive power set-point sent by the TSO. As far as the synthetic inertia experimentation is concerned, the capability of the different involved technologies (battery storage and wind turbines) in providing efficiently a Rate of Change of Frequency (ROCOF)-proportional power response will be tested. So far, inertia provision from wind power plant is specified by the Italian Grid Code [7] and it could be updated taking into account the experimentation results.

In Pietragalla, syntethic inertia provision is demanded to the storage system. For this purpose, during the course of the Osmose project, a Synthetic Inertia Control Device has been developed to cover the entire service chain, from measuring frequency and ROCOF, to calculating the power set-point, commanding the inverter and registering the final output measurements. The power response can be modified by setting specific customizable parameters. By acting on these parameters, it will be possible to vary the derivative and/or proportional contribution as well as the filtering of the ROCOF measurement for the recognition of typical network events that require frequency regulation. As of now, this device is being integrated in the plant overall control system and real time execution will then follow. Moreover, the plant control system of the EGP plant has been updated to implement and include regulation and control algorithms so to allow AVC testing from both the BESS and wind plant in an integrated way. TSO-plant communication has been laid down to allow direct tele-control of the plants during the AVC experimentation, through the transmission of set-points and commands elaborated by the TSO based on the plant output. Analogously, in Vaglio the E2i plant control system has been redesigned as to implement the new algorithms that would allow AVC experimentation. In this case, the reactive power contribution will be only provided by wind turbine generators. TSO-plant communication is here present as well. As far as the synthetic inertia experimentation in Vaglio is concerned, its focus will be the technical characterization of the inertial response provided by a single wind turbine generator. During the Osmose project, following the given desiderata, the wind turbine manufacturer has integrated the Synthetic Inertia control within the firmware of the converter control and will validate it in a reduced bench scale test. Followingly, such modifications will be implemented on a single wind turbine in the Vaglio wind farm and synthetic inertia provision will be tested locally.

\section{ACKNOWLEDGMENTS}

The present paper is realized by the members of the OSMOSE consortium, funded by the H2020 programme under the call: LCE4-2017- Low carbon electricity (project ID 773406).

\section{REFERENCES}

[1] Skamarock, et al. Powers, G. A Description of the Advanced Research WRF Version 3. 2008, doi:10.5065/D68S4MVH.

[2] Pielke, R.A et al. A comprehensive meteorological modeling systemRAMS. Meteorol. Atmospheric Phys. 1992, 49, 69-91, doi:10.1007/BF01025401.

[3] M.N. Wright, A. Ziegler, "Ranger: A fast implementation of random forests for high dimensional data in C++ and R.”, Journal of Statistical Software, 2017, 77(1). https://doi.org/10.18637/jss.v077.i01

[4] L. Breiman, "Random Forests, Machine Learning.", 2001, 45 (1), 5-32, https://doi.org/10.1023/A:1010933404324

[5] M.N. Wright, A. Ziegler, "Ranger: A fast implementation of random forests for high dimensional data in C++ and R.”, Journal of Statistical Software, 2017, 77(1). https://doi.org/10.18637/jss.v077.i01

[6] D. Villacci et al. "Congestion Management in Italian HV grid using novel Dynamic Thermal Rating methods: first results of the H2020 European project Osmose", 2020, pp. 1-3.

[7] Terna S.p.a, annex 17 of the Italian Grid Code: "CENTRALI EOLICHE - Condizioni generali di connessione alle reti AT Sistemi di protezione regolazione e controllo", pp. 32. 